\title{
CAPÍTULO VIII \\ EVALUACIÓN DE LA CALIDAD MICROBIOLÓGICA \\ DE AGUA POTABLE DE DOS PUEBLOS PALAFÍTICOS DE LA CIÉNAGA GRANDE DE SANTA MARTA
}

\section{José Luis Lugo}

Magíster en Ingeniería Ambiental, Investigador de los grupos de investigación: Grupo Udecino de Investigación Ambiental Universidad de Cundinamarca, y Suelo, Ambiente y Sociedad Universidad del Magdalena. Emails: jllugo@ucundinamarca.edu.co

EIkyn Rafael Lugo

Magister en Economía, Docente Investigador del grupo Desoge de la Corporación Universitaria Minuto de Dios Uniminuto. e-mail: elkyn.lugo@uniminuto.edu.co y elkynlugo@gmail.com.

\section{Sandra Bibiana Vargas}

MSc Environmental Sciences, Investigador del grupo de investigación, Grupo Udecino de Investigación Ambiental Universidad de Cundinamarca sbvargas@ucundinamarca.edu.co

\section{Luis Fernando Landazury-Villalba}

$\mathrm{PhD}$ (c) en Administración de Negocios. Profesor investigador, Corporación Universitaria Minuto de Dios-UNIMINUTO, Barranquilla, Colombia. 1landazuryv@ uniminuto.edu.co, https://orcid.org/0000-0003-1190-4171

\section{Jonathan Castro Mercado}

Especialista en Estadística Aplicada; Docente Catedrático en la Corporación Universitaria Minuto de Dios Uniminuto y en la Institución Universitaria de Educación Superior (ITSA).E-mail: uajcastromercado@hotmail.com y jcastromerc@gmail.com

\section{Resumen}

El presente trabajo tuvo como objetivo evaluar la calidad microbiológica del agua de consumo de los habitantes de dos pueblos palafíticos de la Ciénaga Grande de Santa Marta: Nueva Venecia y Buena Vista. En este estudio se realizaron 4 muestreos de calidad de agua potable (dos en época seca y dos en época lluviosa) en 38 puntos de muestreos ubicados en tanques de almacenamiento de agua y sistemas de distribución de los pueblos estudiados. Los parámetros de calidad de agua que se determinaron fueron: Coliformes totales y fecales, sólidos disueltos totales, $\mathrm{pH}$ y conductividad. Se encontró que los coliformes totales y fecales excedieron los límites permisibles de calidad de agua potable estipulados en la normatividad colombiana, oscilando entre 1 x $10^{4}$ y 130 x $10^{4}$ UFC/100 $\mathrm{ml}$, y entre $62 \times 10^{4}$ y $462 \times 10^{4} \mathrm{UFC} / 100 \mathrm{ml}$, respectivamente; mientras que el pH (varió de 6,7 a 9) y conductividad (el valor máximo fue 700 microsiemens $/ \mathrm{cm}$ ) cumplieron con la normatividad colombiana, y los sólidos disueltos totales no se encuentran regulados (entre 20 y $2198 \mathrm{mg} / \mathrm{L}$ ). En un Análisis de varianza multivariado se determinó que la época climática tiene un efecto significativo sobre coliformes totales y fecales (Pvalor $=0,0000)$; y se no se encontró asociación entre sólidos disueltos y coliformes totales ( $\mathrm{P}$-valor= 0,7206$)$, 
en el caso de los coliformes fecales esta relación estadística fue muy leve ( $\mathrm{P}$ valor $=0,048)$. Finalmente, se concluye que el agua de consumo de los habitantes de estos pueblos presenta un alto grado de contaminación bacteriana indicando un alto riesgo sanitario; por lo que, para mejorar la calidad de vida de la comunidad estudiada se hace necesario el diseño y construcción de instalaciones sanitarias que faciliten la evacuación de excretas, el manejo de residuos sólidos y principalmente la potabilización del agua.

Palabras clave: Calidad de agua potable, indicadores microbiológicos, pueblos palafíticos, Ciénaga Grande de Santa Marta.

\title{
EVALUATION OF THE MICROBIOLOGICAL QUALITY OF DRINKING WATER OF TWO PALATITIC VILLAGES OF THE CIÉNAGA GRANDE DE SANTA MARTA
}

\begin{abstract}
The purpose of this work was to evaluate the microbiological quality of drinking water of the inhabitants of two palatitic villages of the Ciénaga Grande de Santa Marta: Nueva Venecia and Buena Vista. In this study, 4 samplings were carried out (two in the dry season and two in the rainy season) in 38 sampling points located in water storage tanks and distribution systems of the villages studied. The water quality parameters determined were: Total and fecal coliforms, total dissolved solids, $\mathrm{pH}$ and conductivity. It was found that total and fecal coliforms exceeded the permissible drinking water quality limits stipulated in the Colombian regulations, ranging between $1 \times 10^{4}$ and $130 \times 10^{4} \mathrm{CFU} / 100 \mathrm{ml}$, and between $62 \times 10^{4}$ and $462 \times 10^{4} \mathrm{CFU} / 100 \mathrm{ml}$, respectively; while the $\mathrm{pH}$ (varied from 6.7 to 9) and conductivity (the maximum value was 700 microsiemens / cm) complied with the Colombian regulations, and the total dissolved solids are not regulated (between 20 and $2198 \mathrm{mg} / \mathrm{L}$ ). In a multivariate analysis of variance, it was determined that the climatic season has a significant effect on total and fecal coliforms $(\mathrm{P}-\mathrm{value}=0.0000)$; and there was no association between dissolved solids and total coliforms (P-value $=0.7206$ ), regarding to fecal coliforms the statistical relationship was very slight $(\mathrm{P}$-value $=0.048)$. Finally, it is concluded that the drinking water of the inhabitants of these villages presents a high degree of bacterial contamination indicating a high sanitary risk; Therefore, in order to improve the quality of life of the studied community, it is necessary to design and build sanitary installations to facilitate the evacuation of excreta, the management of solid waste and mainly the purification of water.
\end{abstract}

Keywords: Quality of drinking water, microbiological indicators, palatitic villages, Ciénaga Grande de Santa Marta.

\section{Capítulo resultado de un proyecto de investigación culminado.}




\section{Introducción}

La contaminación microbiológica del agua por factores antropogénicos como la presencia de aguas residuales y materia fecal de origen humano y animal, así como la contaminación por causas naturales, como las escorrentías de aguas pluviales, representan un gran desafío para la salud pública (Sharma, 2018). Una variedad relativamente pequeña de virus y bacterias entéricas, así como protozoos y helmintos son agentes microbiológicos que ponen en riesgo la salud humana, situación que se agrava con malas prácticas de saneamiento e higiene (Katukiza, Ronteltap, Van der Steen, Foppen, \& Lens, 2014). Salmonella, Shigela, Campilobacter, Gardia, Cryptosporidium y agentes virales son los patógenos comúnmente asociados a brotes de enfermedades transmitidas por el agua, estas son comúnmente conocidas como enfermedades gastrointestinales. Sin embargo, también existen nuevos agentes emergentes ya reconocidos, que causan enfermedades como caliciviruses, E. coli 0157:H7, Helicobacter sp., Cyclospora sp., y Taxoplasma sp (Sharma, 2018).

La población más vulnerable tanto física como socioeconómicamente son los más afectados por microorganismos patógenos transmitidos por el agua. Las personas se pueden infectar con estos agentes si consumen el agua directa o indirectamente a través del uso del agua contaminada para lavar ropa, utensilios de cocina, bañarse o inclusive cocinar. Estas prácticas son muy comunes en áreas rurales donde el servicio de agua potable es informal y a veces no regulado (Nguyen, Le, Ribolzi, Rochelle-Newall, \& Sengtaheuanghoung, 2015)

La tasa de muerte, de atenuación y factores de dilución, determinan la concentración de patógenos en asentamientos informales, que frecuentemente se caracterizan por tener infraestructuras deficientes, bajo nivel de servicios sanitarios y acceso limitado a servicios básicos (Katukiza et al., 2014). Aunque la verdadera magnitud del riesgo microbiológico para la salud humana debida a patógenos, especialmente por virus trasmitidos por el agua en países en desarrollo, aún permanece desconocido (Andrade, Dwyer, Neill, \& Hynds, 2018). Generalmente, los casos asociados a enfermedades gastrointestinales transmitidos por el agua no están bien cuantificados, ni siquiera en países desarrollados, debido a fallas en las técnicas de recolección de datos y a la irregularidad en los seguimientos de estudios epidemiológico específicos (Ashbolt, 2015).

Cambios en la hidrología y en la calidad del agua, también son responsables por la aparición de brotes asociados a enfermedades transmitidas por el agua. En países en desarrollo se prevé incrementos en las lluvias e inundaciones debido a al cambio climático, lo cual lleva al lavado de materia fecal de letrinas, lo que puede acarrear la contaminación del agua para consumo a través de ríos, arroyos, lagos, y aguas subterráneas. Este fenómeno se exacerba con el cambio de uso de tierras en áreas tropicales (Nguyen et al., 2015). Se estima que, a escala global, las enfermedades gastrointestinales son responsables de la muerte de 1.5 millones de personas anualmente, especialmente niños en países en desarrollo, y más de la mitad de las muertes registradas son causadas por la falta de acceso al agua limpia y a servicios sanitarios seguros (WHO, 2017).

Se entiende por agua potable el agua cuya calidad no representa ningún riesgo significativo para la salud durante todo el tiempo de consumo, incluyendo los periodos más sensibles durante todo el ciclo de vida y más que una necesidad básica se le considera un derecho universal (Sharma, 2018). Sin embargo, muchas fuentes de agua tanto superficial 
como subterráneas son susceptibles de contaminación y requieren de inversión en plantas de tratamiento de agua, la cual es una limitante en comunidades rurales que son marginadas por el estado y carecen de recursos necesarios para implementar estas tecnologías (Sánchez, 2017), por lo que acuden a tratamientos descentralizados a bajo costo, como lo son los filtros de bioarena.

No obstante, para evaluar la eficiencia de los sistemas de tratamiento se requiere la evaluación de la calidad de agua de manera permanente, así como el monitoreo de la salud de las personas con el fin de establecer programas de mejora en la gestión integral del agua y tener certeza que las características físicas, químicas y microbiológicas del agua no están afectando la salud de las personas.

En el caso de los pueblos palafiticos de la ciénaga Grande de Santa Marta: Nueva Venecia y Buena Vista, que además de carecer de sistemas de saneamiento básico, no existe un seguimiento continuo y específico de las enfermedades de sus habitantes, ni mucho menos de la calidad del agua que consumen estas comunidades. Existe un estudio realizado por Lugo \& Lugo (2018), pero este sólo reportó los datos de calidad de agua de la fuente de captación y no de las viviendas, por lo que se hace pertinente evaluar la calidad de agua de consumo en los hogares con el fin de servir de soporte técnico y científico para la implementación de medidas correctivas para la gestión del agua potable en la comunidad estudiada. Es por ello que el objetivo del presente trabajo fue evaluar la calidad microbiológica del agua de consumo de los habitantes de los pueblos palafíticos de la Ciénaga Grande de Santa Marta.

El área de estudio comprende los corregimientos de Nueva Venecia $\left(10^{\circ} 49^{`} \mathrm{~N}\right.$; $\left.74^{\circ} 34^{\prime} \mathrm{O}\right)$, Buena Vista (1050’N; 74³0’O) ubicados en la ciénaga de pajarales o complejo pajarales (CP), contigua a la ciénaga grande de Santa Marta (CGSM) y las rutas de ingreso de agua potable al pueblo, las cuales van desde el caño de aguas negras (parte noroeste del municipio de sitio nuevo) en Nueva Venecia y desde el rio Aracataca en Buena Vista. Esta ruta atraviesa totalmente la CGSM, el complejo pajarales hasta llegar a su destino. Los municipios anteriormente mencionados son viviendas artesanales construidas en madera sobre el complejo cenagoso, con un total aproximado de 300 y 150 casas respectivamente, y se encuentran a una distancia de 15 minutos en chalupa (Lugo \& Lugo, 2018).

El agua que llega a los pueblos palafíticos es transportada en bongoductos (canoas grandes y profundas que transportan agua) desde la desembocadura del rio Aracataca en Buenavista y desde caño de aguas Negras en Nueva Venecia, hasta cada uno de los corregimientos. Esta agua es suministrada a la comunidad en tanques plásticos sin ningún tipo de tratamiento previo, de esta forma el agua es utilizada para la mayoría de sus actividades cotidianas (consumo, manipulación de alimentos, oficios varios, higiene, entre otros).

\section{Fundamentación teórica}

La evaluación microbiológica del agua potable tiene como objetivo proteger a los consumidores de enfermedades debido a la presencia de agentes patógenos en el agua. Esta evaluación es hasta nuestros días un campo en continuo desarrollo y discusión. El método clásico para esta evaluación microbiológica es el uso de indicadores de contaminación fecal, 
generalmente coliformes fecales y de procesos microbianos, comúnmente coliformes totales (Fewtrell \& Bartram, 2001). Coliformes totales y fecales tienen el potencial de predecir la presencia de organismos patogénicos en el agua (Figueroa, 2010), sin embargo mucha discusión se ha generado acerca de la correlación entre la concentración de indicadores fecales y la concentración o presencia de patógenos (Hofstra, 2011). La organización mundial de la salud (OMS) es una institución global muy activa en el desarrollo de guías y normativas que son adoptadas por la legislación de la mayoría de países en el mundo, estas recomendaciones incluyen el uso de indicadores como coliformes totales y fecales (Figueroa, 2010). Hasta la fecha no se ha realizado la evaluación de la calidad microbiológica en pueblos palafíticos de la ciénaga Grande de Santa Marta, diferente a la desarrollada en este estudio. Sin embargo, este tipo de análisis se ha realizado en áreas rurales como Kampong Ayer, Brunei (Goh, 1991) y el Delta del Mekong en Vietnamita (Grady, Van Nguyen, Van Nguyen \& Blatchley, 2018).

\section{Metodología}

En el diseño de investigación se requirió emplear un enfoque cuantitativo para evaluar la calidad microbiológica de agua potable de los pueblos palafíticos de estudio, con el fin de evaluar la idoneidad que tiene esta agua para el consumo humano, que es fundamentalmente la problemática que se aborda en el presente trabajo. Este estudio es de tipo descriptivo, en el que se procesan datos microbiológicos que fueron tomados en diferentes muestreos donde se recolecta información (tomas de muestreas de agua) mediante guías elaboradas por centro de estudios de Colombia, y esta se analiza con estadísticos descriptivos e inferencial, como se explica a continuación:

\section{Caracterización fisicoquímica y microbiológica del agua de consumo humano}

La caracterización fisicoquímica y microbiológica de las fuentes de abastecimiento y depósitos de almacenamiento del agua de consumo del área de estudio se realizó por medio de la selección de varios puntos de muestreo, los cuales se determinaron mediante la siguiente ecuación de muestreo aleatorio simple:

$$
n=\frac{n^{\prime}}{1+\left(\frac{n^{\prime}}{N}\right)}
$$

Dónde: $\mathrm{n}=$ número de muestras, $\mathrm{N}=$ población, $\mathrm{n}=$ varianza de la muestra $\left(\mathrm{s}^{2}\right) /$ varianza de la población $\left(\delta^{2}\right)$, la varianza de la muestra está en función de la confiabilidad y la varianza de población en función del error estándar (se). En este estudio se trabajó con una confiabilidad del 95\% $(0,95)$ y un error estándar del 5\% $(0,05)$. Realizando los cálculos se obtuvo una muestra de 18 casas para Nueva Venecia y 16 en Buena vista. En total se ubicaron 38 puntos de muestreo a lo largo de la red de distribución y en los puntos de almacenamiento, tanto en las casas como en las albercas de almacenamiento (Ver Tabla 1). 
Los puntos de muestreo fueron codificados de la siguiente manera: un código para identificar el lugar o dispositivo donde se tomó la muestra y un número único para identificar cada sitio. Específicamente se usaron: [NV]: Casa de Nueva Venecia, [BV]: Casa de Buena Vista, $\left[\mathrm{NV}_{\text {Bong }}\right]$ : Bongoducto de Nueva Venecia, $\left[\mathrm{BV}_{\text {Bong }}\right]$ : Bongoducto de Buena Vista, $\left[\mathrm{NV}_{\mathrm{Alb}}\right]$ : Alberca de Nueva Venecia, $\left[\mathrm{NV}_{\text {Capt }}\right]$ : Captación de Nueva Venecia (punto de captación ubicado en el caño Aguas Negras).

Tabla 1: Localización de los puntos de muestreo

\begin{tabular}{cc}
\hline Puntos de muestreo & Ubicación \\
\hline A & Sitio Nuevo (Caño de Aguas Negras). \\
\hline B & Nueva Venecia (18 casas). \\
\hline C & Buena Vista (16 casas). \\
\hline D & Bongoductos. (1 en Nueva Venecia y 1 en Buena Vista) \\
\hline E & Albercas donde depositan el agua los bongoductos (1 en nueva Venecia) \\
\hline
\end{tabular}

Fuente: Elaboración propia (2019).

\section{Frecuencia de muestreo}

Los 38 puntos de puntos de monitoreo seleccionados se sometieron a 4 muestreos continuos, durante los meses de marzo, abril, junio y julio de 2012; que correspondieron a temporada seca y precipitación. La recolección de las muestras en todos los puntos de monitoreo determinados fue desarrollada con base a la Guía para el monitoreo de vertimientos, aguas superficiales y subterráneas elaborada por el Instituto de Hidrología, Meteorología y Estudios Ambiéntales (IDEAM) de Colombia.

\section{Parámetros de evaluación y análisis de laboratorio}

Los análisis de laboratorio se realizaron en el laboratorio de calidad de aguas de la Universidad del Magdalena, teniendo en cuenta lo establecido en los Standard Methods for examination of water and wastewater. La determinación de coliformes totales y fecales, se realizó utilizando la técnica de filtración por membrana (referencia 9222 B); y. los sólidos disueltos totales fueron estimados teniendo en cuenta la determinación de sólidos totales (2540B, Gravimétrico $103-105^{\circ} \mathrm{C}$ ) y de sólidos suspendidos totales (Standard Methods 2540D, Gravimétrico $103-105^{\circ} \mathrm{C}$ ). Todos los parámetros analizados en el laboratorio se evaluaron por duplicado, reportando el promedio obtenido. Finalmente, para evaluar la calidad microbiológica y fisicoquímica del agua se procedió a comparar los resultados obtenidos con la legislación vigente colombiana de calidad del agua potable (Resolución 2115 de 2007). 


\section{Análisis Estadísticos}

Se procedió a realizar un análisis de varianza multifactorial y descriptivo de los datos obtenidos. El análisis de varianza multifactorial se realizó para determinar la influencia de la época climática (húmeda y seca) y los sólidos disueltos en los datos obtenidos de coliformes totales y fecales en los dos pueblos palafíticos estudiados, así como la interacción entre estos parámetros mencionados. Para ello, se utilizó el software estadístico STATGRAPHICS Centurión versión 16.1.15.

\section{Resultados}

Los resultados de la caracterización de la calidad de agua de consumo humano en los 4 muestreos realizados en los pueblos palafíticos de la Ciénaga Grande de Santa Marta durante las épocas secas ( 2 muestreos) y lluviosas (2 muestreos) se analizan de manera individual por parámetro en el transcurso de este ítem.

\section{Coliformes totales y Coliformes fecales}

La prevalencia de coliformes totales y fecales ( $>0 \mathrm{UFC} / 100 \mathrm{ml}$ de agua) en todas las muestras de agua, tanto en temporada húmeda como seca fue del 100\% ( $\mathrm{n}=184$ muestras). Esta información puede observarse en la Figura 1. Los promedios de coliformes fecales fueron de $8 \times 10^{4} \mathrm{UFC} / 100 \mathrm{ml}$ en época seca, y de $43 \times 10^{4} \mathrm{UFC} / 100 \mathrm{ml}$ en época húmeda, siendo estimados el valor máximo, el valor mínimo y desviación estándar, de $130 \times 10^{4}$, $1 \times 10^{4}$ y $32 \times 10^{4} \mathrm{UFC} / 100 \mathrm{ml}$, respectivamente. En el caso de coliformes totales, se obtuvieron promedio, mínimo y máximo de $62 \times 10^{4}, 462 \times 10^{4}$, y 1 x $10^{4} \mathrm{UFC} / 100 \mathrm{ml}$, respectivamente. Estos resultados son muy preocupantes porque indican alto grado de riesgo de consumo de agua, ya que los niveles de coliformes fecales y totales están en el orden de $10^{4} \mathrm{UFC} / 100 \mathrm{ml}$.

El planteamiento de la hipótesis de estadística inferencial para el indicador microbiológico Coliformes totales y fecales con relación a la época (lluviosa o seca) y a los sólidos disueltos es el siguiente: $\mathrm{H}_{\text {nula }}=$ Las épocas (húmeda y seca) no influyen en los calidad microbiológica del agua (Coliformes totales o fecales). $\mathrm{H}_{\text {alternativa }}=$ Las épocas (húmeda $\mathrm{y}$ seca) influyen en la calidad microbiológica del agua (Coliformes totales o fecales). En la Tabla 2 se resume el ANOVA multifactorial realizado en el software STATGRAPHICS Centurión versión 16.1.15. 

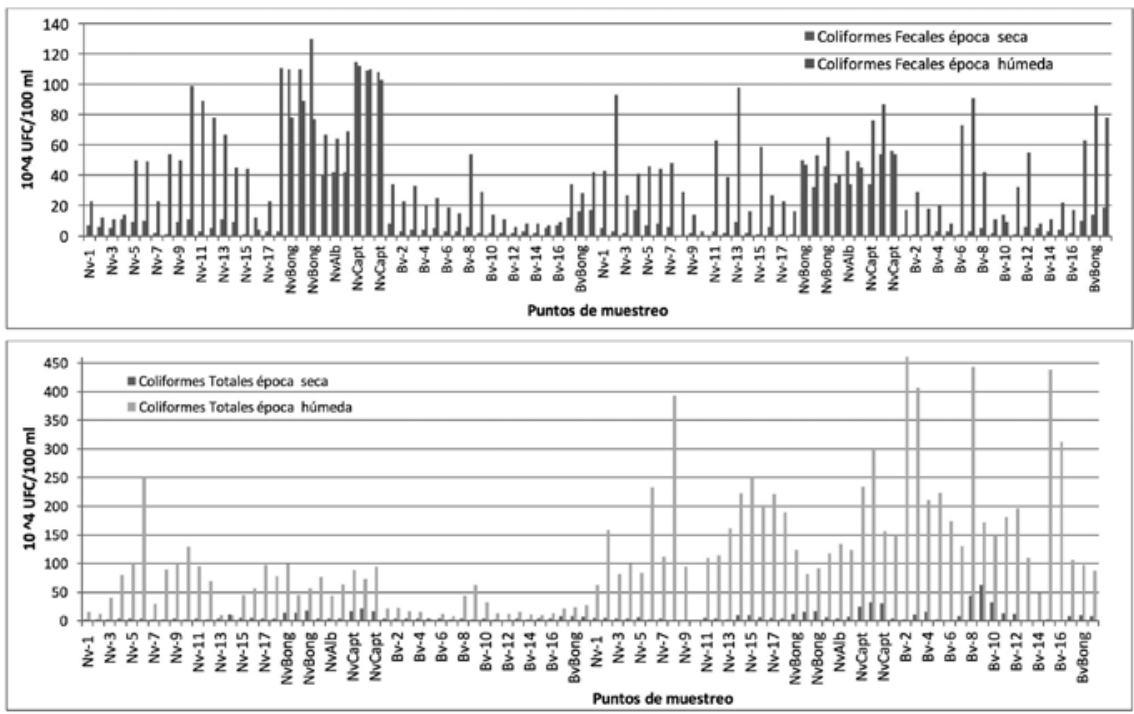

Figura 1. Variación espacio temporal de la calidad microbiológica de agua potable en el área de estudio.

Fuente: Elaboración propia (2019).

Tabla 2. Resultados de Análisis de Varianza multifactorial para Coliformes Totales y fecales

\begin{tabular}{ccc}
\hline Fuente & Coliformes totales & Coliformes fecales \\
\hline \multirow{2}{*}{ EFECTOS PRINCIPALES } & Valor-P & Valor-P \\
\hline A:Epoca & 0 & 0 \\
\hline B:Sólidos Disueltos & 0,7206 & 0,048 \\
\hline
\end{tabular}

Fuente: Elaboración propia (2019).

Los resultados obtenidos muestran que la época climática según el valor-P $(0,0000)$ tiene un efecto estadísticamente significativo sobre coliformes totales y fecales; mientras que en el caso de los sólidos disueltos no se encontró un asociación sobre los coliformes totales $(\mathrm{P}$-valor $=0,7206)$, y muy leve en el caso de los coliformes fecales $(\mathrm{P}$ valor $=0,048)$.

\section{PH, sólidos disueltos y conductividad}

El parámetro $\mathrm{pH}$ no presentó variación sustancial en su comportamiento a lo largo del tiempo y con respecto a las épocas del año, estando en el rango entre 6.5 y 9 unidades, 
como lo indica la resolución 2115 del 2007 del Ministerio de Ambiente y Desarrollo Sostenible. La conductividad tampoco sobrepasó el nivel máximo permisible expuesto en la normatividad colombiana en ningún caso (1000 microsiemens/cm), siendo el valor mayor reportado de 700 microsiemens/cm. En cuanto a los sólidos disueltos oscilaron entre 20 y $2198 \mathrm{mg} / \mathrm{L}$, y no se tiene un valor de referencia que pueda indicar su grado nocividad para la salud humana; sin embargo, se sugiere desarrollar un análisis más completo sobre la composición química de estos sólidos con el fin de evaluar su nivel de riesgo para la salud humana en el consumo de agua de los pueblos estudiados.

\section{Discusión}

La contaminación fecal se puede originar de muchas fuentes, incluido el agua residual de asentamientos humanos (Amenu, Spengler, Markemann, \& Zárate, 2014). Las concentraciones de Coliformes totales y fecales en asentamientos de la CGSM son influenciadas principalmente por la descarga directa de los residuos domésticos de las mismas poblaciones, las cuales no cuentan con un sistema de saneamiento básico adecuado para la eliminación de los desechos (Huda et al., 2018). En este caso, el tamaño de la población es un factor determinante en la cantidad de residuos líquidos y sólidos generados, ya que su disposición afecta significativamente la calidad sanitaria del cuerpo de agua (Narváez, Gomez \& Acosta, 2008).

El análisis de varianza multifactorial mostró un efecto estadísticamente significativo entre estado de tiempo climatológico y la presencia de coliformes totales y fecales, lo cual indica que los puntos de muestreo se encuentran influenciados por el aporte de vertimientos domésticos provenientes de las poblaciones y por las condiciones ambientales (Narváez et al., 2008; Zamora-Bornachera, Narváez-Barandica \& Londoño-Díaz, 2007). De igual manera, los aportes contaminantes son asociados a las precipitaciones, dado que es una contaminación difusa que arrastra contaminantes en los cuerpos de agua (Gershunov, Benmarhnia \& Aguilera, 2018; Yu, Xu, Wu \& Zuo, 2016).

En Colombia, al igual como sucede en otros países de América Latina, la ausencia de un sistema de manejo eficiente de excretas, tratamiento de agua y otros residuos domésticos han conllevado a la contaminación de los cuerpos de agua (González, Torres, \& Chiroles, 2003). En este caso, con repercusiones a nivel ambiental y sanitario, ya que en todos los muestreos se sobrepasaron los valores de Coliformes totales y fecales establecidos en la normativa colombiana (0 UFC/ $100 \mathrm{~mL}$ ) para consumo humano, estando los habitantes expuestos a la adquisición de enfermedades gastrointestinales debido a la carencia de sistemas de tratamiento de agua potable y prácticas inadecuadas de higiene, que incrementan el riesgo de propagación de enfermedades asociadas con el consumo de agua contaminada.

La carga microbiana depende de factores ambientales como la lluvia y la radiación solar. En eventos de altas precipitaciones, como el año 2005, los sedimentos de los ríos y la tierra son arrastrados hasta los cuerpos de agua donde se resuspenden los microorganismos y aumentan su concentración (Amenu et al., 2014). En el palafito Nueva Venecia se encontraron las máximas cargas durante el monitoreo, probablemente por la influencia del río Magdalena, que cuenta con grandes fuentes de contaminación, a través del caño Aguas Negras. 
Por otra parte, los sólidos disueltos no presentaron un efecto estadísticamente significativo sobre Coliformes Totales, y muy leve en el caso de coliformes fecales; lo que puede ser explicado porque la contaminación microbiológica depende en sí de las heces fecales presentes en el agua como producto de actividades antropogénicas y no de sales, que en sí son composiciones naturales del agua, la cual en el planeta no se encuentra pura, a excepción que se le haga un tratamiento físico o químico avanzado.

Teniendo en cuenta lo anterior, se puede concluir que no existe una relación entre los sólidos disueltos y la variación el comportamiento de los microorganismos, los cuales sí podrían estar influenciados por otros parámetros que no fueron objeto de estudio de esta investigación, además no se ha encontrado información teórica que permita relacionar estos dos parámetros.

\section{Conclusiones}

El agua de consumo de los habitantes de estos pueblos está contaminada con microorganismos patógenos que deterioran la salud humana, ya que en los análisis de laboratorio realizados a las muestras se encontró que todos los puntos de muestreo evaluados superaron los límites permisibles por la normatividad colombiana, la cual establece 0 UFC/100 ml para Coliformes Totales y Fecales. Los valores obtenidos de estos indicadores microbiológicos analizados en el área de estudio sobrepasaron excesivamente el criterio de calidad de agua potable, oscilando los Coliformes Fecales entre 1 x $10^{4}$ y 130 x $10^{4} \mathrm{UFC} / 100 \mathrm{ml}$, y los Coliformes Totales entre 1 x $10^{4}$ y $462 \times 10^{4} \mathrm{UFC} / 100 \mathrm{ml}$; lo cual indica que el agua es de muy mala calidad y representa una alta probabilidad de contracción de enfermedades asociadas a patógenos contenidos en el agua que consumen los habitantes de los dos pueblos palafíticos estudiados.

Por lo que, para mejorar la calidad de vida de los habitantes de estos pueblos se hace necesario el diseño y construcción de instalaciones sanitarias que faciliten la evacuación de excretas, el manejo de residuos sólidos y sobre todo la potabilización del agua. Por otro lado, en el análisis de varianza multifactorial realizado a los datos obtenidos en los muestreos se encontró que la época climática influye en la concentración de microorganismos patógenos, es decir, la concentración de microorganismos aumenta en la época lluviosa con respecto a la seca.

Finalmente, el pH tuvo un comportamiento estable a lo largo de los muestreos, oscilando entre 6,7 y 9 unidades, cumpliendo así con la normatividad Colombiana aplicable para estos casos. En cuanto a la conductividad eléctrica, aunque tuvo una tendencia a aumentar con respecto a la época climática, es decir, incrementó en la época lluviosa, no sobrepasó los límites establecidos por la Resolución 2115 del 2007 emanada por el Ministerio de Ambiente y Desarrollo Sostenible de Colombia.

\section{Referencias bibliograficas}

Amenu, K., Spengler, M., Markemann, A., \& Zárate, A. V. (2014). Microbial quality of water in rural households of Ethiopia: Implications for milk safety and public health. Journal of Health, Population and Nutrition, 32(2), 190-197. 
Andrade, L., Dwyer, J. O., Neill, E. O., \& Hynds, P. (2018). Surface water flooding, groundwater contamination, and enteric disease in developed countries: A scoping review of connections. Environmental Pollution, 236, 540-549.

Ashbolt, N. J. (2015). Microbial Contamination of Drinking Water and Human Health from Community Water Systems. Current Environmental Health Reports, 2(1), 95-106.

Fewtrell, L., \& Bartram, J. (2001). Water quality: guidelines, standards \& health. Cornwall: IWA publishing.

Figueras, M., \& Borrego, J. (2010). New perspectives in monitoring drinking water microbial quality. International journal of environmental research and public health, 7(12), 4179-4202.

Gershunov, A., Benmarhnia, T., \& Aguilera, R. (2018). Human health implications of extreme precipitation events and water quality in California, USA: a canonical correlation analysis. The Lancet Planetary Health, 2, S9.

Goh, K. (1991). Garbage Problems of a Water Settlement, Kampong Ayer, Brunei Darussalam. Journal of Environmental Systems, 21(1), 85-99.

González, M., Torres, T., \& Chiroles, S. (2003). Calidad microbiológica de aguas costeras en climas tropicales. Revista Cuba, medio ambiente y desarrollo, 4, 1-5.

Grady, C., Van Nguyen, K., Van Nguyen, T., \& Blatchley, E. (2018). Who is being left behind? An analysis of improved drinking water and basic sanitation access in the Vietnamese Mekong Delta. Journal of Water. Sanitation and Hygiene for Development, 8(3), 508-519.

Hofstra, N. (2011). Quantifying the impact of climate change on enteric waterborne pathogen concentrations in surface water. Current Opinion in Environmental Sustainability, 3(6), 471-479.

Huda, T. M. N., Schmidt, W. P., Pickering, A. J., Mahmud, Z. H., Islam, M. S., Rahman, M. S., ... Biran, A. (2018). A cross sectional study of the association between sanitation type and fecal contamination of the household environment in rural Bangladesh. American Journal of Tropical Medicine and Hygiene, 98(4), 967-976.

Katukiza, A. Y., Ronteltap, M., Van der Steen, P., Foppen, J. W. A., \& Lens, P. N. L. (2014). Quantification of microbial risks to human health caused by waterborne viruses and bacteria in an urban slum. Journal of Applied Microbiology, 116(2), 447-463.

Lugo, J., \& Lugo, E. (2018). Beneficios socio ambientales por potabilización del agua en los pueblos palafiticos de la ciénaga grande de Santa Marta-Colombia. Revista UDCA Actualidad \& Divulgación Científica, 21(1), 259-264.

Narváez, S., Gomez, M., \& Acosta, J. (2008). Coliformes termotolerantes en aguas de las poblaciones costeras y palafiticas de la Ciénaga Grande de Santa Marta, Colombia. Acta Biológica Colombiana, 13(3). 
Nguyen, T. M. H., Le, T. P. Q., Ribolzi, O., Rochelle-Newall, E., \& Sengtaheuanghoung, O. (2015). A short review of fecal indicator bacteria in tropical aquatic ecosystems: knowledge gaps and future directions. Frontiers in Microbiology, 6(April), 1-15.

Sánchez, J. (2017). Saneamiento descentralizado y reutilización sustentable de las aguas residuales municipales en México, Sociedad y ambiente, (14), 119-143.

Sharma, B. (2018). Microbes as Indicators of Water Quality and Bioremediation of Polluted Waters, (January), 44-60.

World Health Organization (WHO). (2017). World health statistics 2017: monitoring health for the SDGs, Sustainable Development Goals. Geneva: World Health Organization.

Yu, S., Xu, Z., Wu, W., \& Zuo, D. (2016). Effect of land use types on stream water quality under seasonal variation and topographic characteristics in the Wei River basin, China. Ecological Indicators, 60, 202-212.

Zamora-Bornachera, A., Narváez-Barandica, J., \& Londoño-Díaz, L. (2007). Evaluación económica dela pesquería artesanal de la Ciénaga Grande de Santa Marta y Complejo de Pajarales, Caribe Colombiano, Bol Investig Mar Costeras, 36, 33-48. 